\title{
Designing an Adaptive Web Navigation Interface for Users with Variable Pointing Performance
}

\author{
Aqueasha Martin-Hammond ${ }^{1}$, Foad Hamidi ${ }^{2}$, Tejas Bhalerao ${ }^{2}$, Christian Ortega ${ }^{3}$, Abdullah Ali $^{4}$, \\ Catherine Hornback ${ }^{2}$, Casey Means ${ }^{5}$, Amy Hurst ${ }^{2}$ \\ ${ }^{1}$ Indiana University- \\ Purdue University \\ Indianapolis \\ Indianapolis, IN \\ aqumarti@iupui.edu \\ University of Maryland,
Baltimore County
Baltimore, MD
\{foadhamidi, tejasb1,
cat19,
amyhurst\}@umbc.edu \\ ${ }^{3}$ Texas A\&M \\ University \\ College Station, TX \\ ${ }^{4}$ University \\ of \\ ${ }^{5}$ Rhodes College \\ Memphis, TN \\ christian01@tamu \\ Washington \\ .edu \\ Seattle, WA \\ xyleques \\ meaac- \\ 16@rhodes.edu \\ @uw.edu
}

\begin{abstract}
Many online services and products require users to point and interact with user interface elements. For individuals who experience variable pointing ability due to physical impairments, environmental issues or age, using an input device (e.g., a computer mouse) to select elements on a website can be difficult. Adaptive user interfaces dynamically change their functionality in response to user behavior. They can support individuals with variable pointing abilities by 1) adapting dynamically to make element selection easier when a user is experiencing pointing difficulties, and 2) informing users about these pointing errors. While adaptive interfaces are increasingly prevalent on the Web, little is known about the preferences and expectations of users with variable pointing abilities and how to design systems that dynamically support them given these preferences.
\end{abstract}

We conducted an investigation with 27 individuals who intermittently experience pointing problems to inform the design of an adaptive interface for web navigation. We used a functional high-fidelity prototype as a probe to gather information about user preferences and expectations. Our participants expected the system to recognize and integrate their preferences for how pointing tasks were carried out, preferred to receive information about system functionality and wanted to be in control of the interaction. We used findings from the study to inform the design of an adaptive Web navigation interface, PINATA that tracks user pointing performance over time and provides dynamic notifications and assistance tailored to their specifications. Our work contributes to a better understanding of users' preferences and expectations of the design of an adaptive pointing system.

\section{CCS Concepts}

- Human-centered computing Pointing - Human-centered computing Accessibility design and evaluation methods

\section{Keywords}

Permission to make digital or hard copies of all or part of this work for personal or classroom use is granted without fee provided that copies are not made or distributed for profit or commercial advantage and that copies bear this notice and the full citation on the first page. Copyrights for components of this work owned by others than ACM must be honored. Abstracting with credit is permitted. To copy otherwise, or republish, to post on servers or to redistribute to lists, requires prior specific permission and/or a fee. Request permissions from Permissions@ acm.org.

W4A '18, April 23-25, 2018, Lyon, France

(C) 2018 Association for Computing Machinery.

ACM ISBN 978-1-4503-5651-0/18/04 ..\$15.00

https://doi.org/10.1145/3192714.3192818
Adaptive Interfaces; Assistive Technology; Inclusion; Pointing Problems; Web Navigation.

\section{INTRODUCTION}

For individuals who experience temporary, intermittent, or gradual changes in pointing ability due to a physical impairment, environmental issues or age, using an input device to interact with user interface elements can be difficult. Furthermore, these challenges might be experienced differently at different points in time. For example, an individual with early stage Parkinson's disease may experience significant changes in their ability to control the computer mouse depending on medication regimen or time of day[28, 34]. A younger adult's ability to control a mouse may change depending on sleep schedule, excitement, or caffeine use $[26,27]$. An older adult user may experience changes in hand mobility due to the natural aging process [30]. The individuals in these examples are experiencing mild and infrequent difficulties and might not be aware of their consistent occurrence. Furthermore, these individuals may not have been diagnosed with motor impairment and may not identify as assistive technology users. They may be unaware of these changes, or find it difficult to identify assistive technology solutions that can support their dynamically changing needs, leading to frustrating experiences and further impeding their computer use $[9,27,28,34]$.

Adaptive user interfaces can monitor and dynamically change their appearance and functionality based on user behavior $[16,19$, 31]. They provide an opportunity to support users who experience changes in pointing ability by 1) providing feedback to users about their variable pointing performance, and 2) by adapting system functionality dynamically to make pointing tasks easier in case of difficulties. They are becoming increasingly prevalent, especially on the Web where many of the most popular websites, such as Facebook, Google, Amazon and YouTube, utilize them to better engage users. Despite these opportunities, little is known about the preferences and expectations of such systems from users with variable pointing performance, especially in the context of web navigation.

We conducted a study to better understand user preferences and expectations of adaptive interfaces that support changing pointing ability. We used an interactive prototype as a technology probe [13] and conducted semi-structured interviews with 27 individuals who experience diverse challenges with hand-held input devices. We used the probe to demonstrate notifications, mechanisms to inform the user about their pointing performance, and assistance activation modes, mechanisms to activate interface modification to assist with pointing tasks. We found that regardless of their age

This is the author's manuscript of the article published in final edited form as:

Martin-Hammond, A., Hamidi, F., Bhalerao, T., Ortega, C., Ali, A., Hornback, C., ... Hurst, A. (2018). Designing an Adaptive Web

Navigation Interface for Users with Variable Pointing Performance. In Proceedings of the Internet of Accessible Things (pp. 31:1-31:10).

New York, NY, USA: ACM. https://doi.org/10.1145/3192714.3192818 
and ability, participants were positive about personalized support from an adaptive interface but wanted to be in control of the interaction. They expected the system to take time to adapt to their specific performance and wanted it to avoid introducing unpredictable changes early on. Additionally, they wanted to be informed about system functionality and be included in the system's decision-making process.

We used these insights to design an adaptive interface, Pointing Interaction Notifications and AdapTAtions (PINATA), for web navigation for users who experience pointing difficulties. PINATA can track a user's pointing performance, provide different forms of notifications in response to pointing errors and deploy assistance in the form of a bubble cursor that changes size dynamically in response to user performance. Through a preferences page, end users can specify and the amount and type of information and assistance they want to receive.

In this paper, we present the results of the user study in detail and describe how it informed PINATA's functionality. The contribution of the paper is twofold: it describes a novel adaptive web navigation interface for users who are experiencing pointing difficulties and it describes user preferences and expectations of these systems and how they informed the interface design.

\section{RELATED WORK}

Our work is informed by prior research on the design of adaptive user interfaces and the recognized factors that affect their acceptance. In this section, we discuss users who experience changes in pointing ability, the diversity of pointing abilities, and challenges faced by this population when using input devices to interact with computers. We then discuss prior work on adaptive techniques and systems that support dynamic adaption in response to changing user needs. Finally, we discuss well-documented challenges in designing adaptive interfaces, and how our work contributes to this knowledge.

\subsection{Experiencing Variable Pointing Performance}

Change in perception, cognition and ability is an essential and life-long part of human life. Generally speaking, humans tend to develop these skills in early life, and they degrade with age at varying rates $[5,9,30]$. Changes in hand mobility can lead to negative changes in pointing ability, such as difficulty clicking on targets, pressing mouse buttons, performing drag and drop actions, and finding the cursor. Changes in pointing ability can occur due to age $[3,12,17,18,26,35,38,41]$, a temporary or sustained disability [32, 37, 39] or medical conditions such as Parkinson's, arthritis, or fatigue $[17,30]$. These changes can make it difficult for individuals to use a computer $[3,14,17,30]$ and may limit their technology use.

In some cases, individuals are unaware of the cause of their input errors [27, 28] or changes in their abilities [9]. Moffatt's studies of pen-based menu selection tools revealed that users of their system were often unaware of the cause of their difficulty selecting menu items and why unintended menu items opened [28]. Other individuals are well aware of changes in pointing ability, however these changes occur with unpredictable frequency and severity. It can, therefore, be difficult for them to assess and address their accessibility needs and to find assistive technology solutions that support their dynamically changing needs $[34,36]$.

\subsection{Supporting Variable Pointing with Automation}

In the last decade, adaptive (or personalized) pointing systems have emerged as a beneficial solution for assisting individuals with dynamically changing pointing abilities. These systems provide assistance by changing presentation or behavior based on user input, the state of a system, or both $[16,19,31]$. Because the system can adapt to changing needs, adaptive systems may lessen a user's need to acquire or search for other assistive technology solutions as their needs for pointing support change. These systems can also be helpful for users that do not self-identify as having a motor impairment or are unaware of declines in pointing ability by informing them of changes and automatically providing assistance to minimize frustration.

Prior research on adaptive pointing systems has focused on providing personalized support to individuals, either through 1) building performance assessment techniques or 2) building systems that assist with pointing tasks. Hurst and colleagues examined ways to automatically detect changes in pointing performance as an individual uses a pointing device to interact with a computer $[14,15]$. Approaches, such as this, serve as a platform on which systems are built to automatically inform users of pointing behaviors or assist users with challenging pointing tasks.

In addition to creating performance assessment techniques, several systems have been designed to provide assistance with pointing challenges that impact target acquisition [12, 40] and target selection $[1,17,20,37,42]$. These systems help improve a user's ability to use an input device to select interface elements (e.g. clicking on buttons, positioning the cursor). Gajos and colleagues alternatively designed SUPPLE, a system to support device-specific interface optimization [7]. Heron and colleagues developed the ACCESS frameworks to allow users to control and adjust different pointing assistances to meet their particular needs [10]. This work has increased knowledge of pointing device usage among different user groups and how assistance techniques designed for specific pointing changes affect participants' performance. However, for users with intermittent or gradual changes who may be unaware of having pointing difficulties due to gradual changes or denial, there is a significant gulf in literature regarding users' perceptions of these systems, preferences for the system's interface design, and adoption of these tools.

\subsection{The Challenges of Designing Adaptive Interfaces}

In general, it is well known that while adaptive systems offer many benefits for assisting users with tasks, there are also tradeoffs to introducing automation and change in interface design [16, 19]. Acceptance of an adaptive system that provides automatic assistance often depends on its accuracy (e.g. does it help the user to successfully complete the task at hand?). However, individual preferences, costs, and benefits can also impact acceptance and should be evaluated early to address user concerns [16, 19]. Poorly designed adaptive systems can violate well-established usability principles, such as consistency [29], making it harder for users to predict, comprehend, and control the system while the system in-turn becomes more distracting and intrusive to user tasks [16]. Horvitz notes that another challenge of introducing automation is that it can be difficult to automatically assess the amount and type of support a user wants [11]. 
Table 1. Participant Demographics: We recruited a total of 27 participants (16 over the age of 55). Of these participants, five had Parkinson's (P1-P5) and one (OA8) was recovering from a stroke ( $\mathrm{P}=$ Parkinson's, $\mathrm{OA}=$ Older Adults, YA= Young Adults). A * represents that a participant self-reported motor impairment. A + represents self-reporting multiple pointing problems.

\begin{tabular}{|l|l|l|c|c|}
\hline \multicolumn{1}{|c|}{ ID } & \multicolumn{1}{|c|}{ Age } & Sex & Weekly Web Use & $\begin{array}{c}\text { Pointing } \\
\text { Problems }\end{array}$ \\
\hline P1* & $55-64$ & M & 5 or more days & + \\
\hline P2* & $55-64$ & F & 5 or more days & + \\
\hline P3* & $55-64$ & M & 5 or more days & + \\
\hline P4* & $65-74$ & M & 5 or more days & + \\
\hline P5* & $65-74$ & F & 5 or more days & + \\
\hline OA1 & $65-74$ & F & $3-4$ days & \\
\hline OA2 & $75-84$ & F & $3-4$ days & + \\
\hline OA3 & $75-84$ & M & 5 or more days & + \\
\hline OA4 & $75-84$ & F & 5 or more days & + \\
\hline OA5 & $65-74$ & F & $1-2$ days & + \\
\hline OA6 & $75-84$ & F & 5 or more days & + \\
\hline OA7 & $65-74$ & F & $<1$ day & + \\
\hline OA8* & $65-74$ & F & 5 or more days & + \\
\hline OA9 & $65-74$ & F & 5 or more days & + \\
\hline
\end{tabular}

\begin{tabular}{|l|l|c|c|c|}
\hline \multicolumn{1}{|c|}{ ID } & \multicolumn{1}{c|}{ Age } & Sex & Weekly Web Use & $\begin{array}{c}\text { Pointing } \\
\text { Problems }\end{array}$ \\
\hline OA10 & $65-74$ & F & 5 or more days & + \\
\hline YA1 & $18-24$ & F & 5 or more days & + \\
\hline YA2 & $18-24$ & M & 5 or more days & \\
\hline YA3 & $25-34$ & F & 5 or more days & + \\
\hline YA4 & $25-34$ & M & 5 or more days & \\
\hline YA5 & $25-34$ & F & 5 or more days & + \\
\hline YA6 & $18-24$ & M & 5 or more days & \\
\hline YA7 & $25-34$ & M & 5 or more days & + \\
\hline YA8 & $18-24$ & M & 5 or more days & + \\
\hline YA9 & $25-34$ & M & 5 or more days & + \\
\hline YA10 & $18-24$ & F & 5 or more days & + \\
\hline YA11 & $18-24$ & F & 5 or more days & + \\
\hline YA12 & $25-34$ & M & 5 or more days & + \\
\hline & & & & \\
\hline
\end{tabular}

There has been significant debate about the role of automation in interface design $[11,33]$. Some have argued for manual user manipulation where others have argued for automated software agents [33], or a combination of both [11]. Horvitz contends that one key barrier to using interface agents is that it can be hard to guess users' goals and needs and therefore argues for a mixedinitiative approach in which agents and users collaborate to achieve a goal [11].

Due to the known challenges of introducing automation into interface design, recently, researchers have started exploring how various factors affect user experience, attitudes and potential acceptance of these systems [22, 23]. In a prior study of adaptive pointing systems, researchers identified factors such as information preferences or trustworthiness that were important to consider when designing these systems [22, 23]. In this study, we extend prior work by understanding the needs of users that experience intermittent or gradual changes in hand mobility and do not currently identify as having an impairment that completely impedes their computer use. We study the factors that impact this unique population's acceptance of adaptive user interfaces that assist with variable pointing performance. We identify this population's preferences and expectations of adaptive pointing solutions with the goal of understanding the broader rationale behind their preferences and expectations. Additionally, we describe how our findings informed the design of an adaptive web navigation interface, PINATA that provides notifications and adaptations to support users with variable pointing performance.

\section{USER PREFERNECES AND EXPECTATIONS OF ADAPTIVE INTERFACES}

We conducted semi-structured interviews with individuals who experienced intermittent or permanent pointing problems. Participants interacted with a functional prototype of the system as a probe [13] to gather insight that focused on user expectations of real-time personalized pointing information, notification delivery, and adaptive pointing assistance activation modes. Interacting with a functional probe is found to be an effective communication and collaboration tool in previous research [13]. In the following sections, we describe our participants, the probe and procedures we utilized in the study. This study was approved by the Institutional Review Board (IRB) at UMBC.

\subsection{Participants}

We recruited 27 participants with diverse abilities to understand a broad range of perspectives regarding pointing information: 12 younger adults ( $18-34$ years), 10 older adults (ages 65 years or older), and 5 adults (ages 55 years or older) with early-stage Parkinson's (See Table 1). At the time of the study, no participants reported impairments that would completely impede their use of a computer. However, across the groups, 23 participants reported experiencing intermittent difficulty when using a pointing device. Most of the participants who had difficulty with pointing tasks adopted manual approaches to cope (e.g. scheduling computer time to avoid peaks in tremors). The five participants with early-stage Parkinson's all experienced intermittent hand tremors that varied depending on time of day or medication regimen, and one older adult reported a motor impairment affecting her hands due to stroke.

Table 2. Common pointing challenges reported by participants (A = All the Time, $S=$ Sometimes $)$

\begin{tabular}{|l|c|c|c|}
\hline & $\begin{array}{c}\text { Parkinson's } \\
(\mathbf{n}=\mathbf{5})\end{array}$ & $\begin{array}{c}\text { Older } \\
\text { Adults } \\
(\mathbf{n}=\mathbf{1 0})\end{array}$ & $\begin{array}{c}\text { Young } \\
\text { Adults } \\
(\mathbf{n}=\mathbf{1 2})\end{array}$ \\
\hline $\begin{array}{l}\text { Unintentional } \\
\text { selection }\end{array}$ & $\mathbf{A}=2, \mathbf{S}=3$ & $\mathbf{A}=0, \mathbf{S}=8$ & $\mathbf{A}=0, \mathbf{S}=10$ \\
\hline Slip off a target & $\mathbf{A}=1, \mathbf{S}=3$ & $\mathbf{A}=1, \mathbf{S}=8$ & $\mathbf{A}=0, \mathbf{S}=3$ \\
\hline $\begin{array}{l}\text { Overshoot or miss } \\
\text { a target }\end{array}$ & $\mathbf{A}=1, \mathbf{S}=3$ & $\mathbf{A}=0, \mathbf{S}=8$ & $\mathbf{A}=0, \mathbf{S}=3$ \\
\hline $\begin{array}{l}\text { Cursor loss from } \\
\text { erratic movement }\end{array}$ & $\mathbf{A}=1, \mathbf{S}=1$ & $\mathbf{A}=3, \mathbf{S}=4$ & $\mathbf{A}=0, \mathbf{S}=6$ \\
\hline
\end{tabular}


Since our study included users who might not have been diagnosed with a condition leading to mobility impairment and who might have experienced temporary or gradual changes in pointing, we asked if participants experienced difficulties identified previously with mouse use [17]. In Table 2, we present the most common pointing problems reported by the participants including: unintentional selection of items on a webpage, slipping off a target such as a menu item, overshooting or missing a target, and erratic movement leading to cursor loss.

\subsection{Materials}

We used a high-fidelity functional prototype in the form of a Chrome Extension as a technology probe [13]. The design of the prototype was informed by past work on designing notifications and adaptive systems for users with diverse pointing abilities [22, 23]. The prototype simulated three types of notifications (Figure 1) and demonstrated one type of assistance which consisted of a zooming functionality that made a webpage's elements larger if pointing errors were detected.

The first notification design, the Bar (Figure 1, A), was an abstract indicator in the form of a red bar that would appear on top of the page when frequency errors were detected. The second notification design, the Bar+ (Figure 1, B), was similar to the bar notification but also included information about the pointing error that occurred and gave the user options to activate an assistance or to ignore the message. The third type of notification, the Dialogue Box (Figure 1, C), consisted of a box that provided information about detected errors and options to activate or ignore assistance.

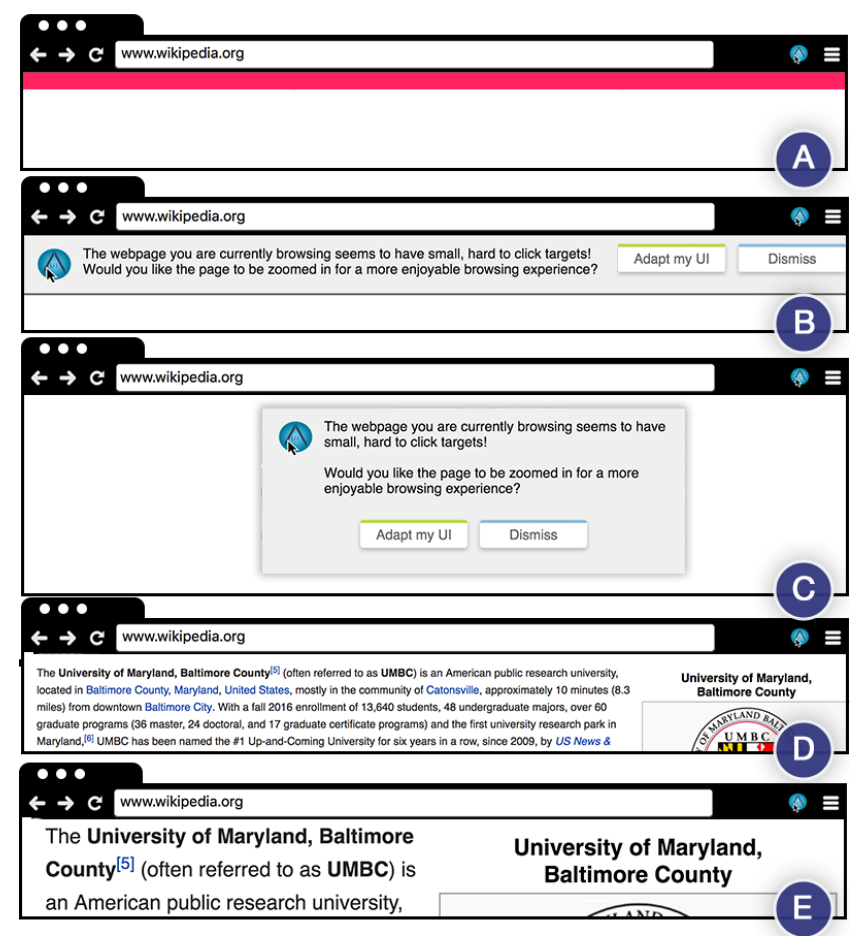

Figure 1. Notification and Activation Options: (A) The Bar notification appears as a thin horizontal bar under the URL field. (B) The Bar+ notification appears on as a horizontal box under the URL field with text and interactive buttons. (C) The Dialog Box appears as a popup alert with text and interactive buttons. (D) Wikipedia page zoomed at $100 \%$ (default viewing size). (E) Wikipedia page at $150 \%$ viewing size after manually zooming in.
We used the different types of notifications and the assistance to illustrate the diverse ways the system might offer pointing information and assistance. While the probe included a listener that captured real-time user performance data (e.g. the duration of clicks), we modified the system so that notifications were deployed regardless of pointing performance when a user clicked on links within the webpage. Participants were not informed that a clicking on the link would trigger a notification. We did this for two reasons. First, we wanted to provide each user with a similar demonstration of how notifications or assistance could be tied to pointing problems without introducing bias. Second, given the exploratory approach of our study, we wanted to provide the flexibility to support users' discussion of other ideas not included in the prototype's design.

We used a zooming mechanism (Figure 1D and 1E) that increased the size of user interface elements to demonstrate the three ways that an assistance could be activated: automatically, where it is deployed in response to an increase in pointing errors; using a mixed-initiative, where it is recommended to the user when errors are detected and activated by them; and manually, where the user activates it themselves.

During the study, participants used a Mac Book Pro computer with an attached mouse. All participants used the same computer and mouse device. To provide a similar experience for all participants, they were not allowed to make adjustments to the appearance or behavior of the pointer. They were however provided with time in the beginning of the study to acquaint themselves with the computer mouse and browser.

\subsection{Data Collection and Analysis Procedures}

For each participant, we first administered a background questionnaire asking about demographics, computer use, pointing performance experience, and current challenges with input devices. To help participants answer questions about challenges with input devices and ensure they were familiar with our terms, we defined each pointing challenge (e.g. unintentional selection, overshooting a target) and provided examples, if requested.

Then we talked with participants about their experience with personalized pointing support. Using the challenges participants listed as pointing problems in the background survey, we provided them with a scenario of how a personalized pointing system might automatically provide them with assistance with the pointing problem. We kept this discussion broad by not focusing on how the assistance might look, but rather on helping the participant understand that the system would track their mouse activity and use that information to provide personalized notification and/or assistance to meet their changing needs.

We next engaged participants in semi-structured interview sessions that on average lasted 60 minutes. Each interview session included the following phases: 1) assessing the importance of notification visibility, 2) identifying pointing information delivery preferences, 3) identifying information needs, and 4) identifying assistance information and activation preference(s). We took detailed handwritten notes of participants' verbal responses and administered several paper questionnaires throughout the study to collect written responses. Participants received four surveys throughout the study each of which was followed by a discussion and an opportunity to elaborate on responses. These questions were informed by prior work and focused on aspects of the designs that could potentially negatively or positively impact their perception of a personalized pointing system [22, 23]. All 
participants interacted with all 3 notification designs and were provided with explanations of all 3 assistance activation modes.

\subsubsection{Four Phases of Data Collection}

Participants engaged in naturalistic browsing tasks while interacting with a Wikipedia page to assess the visibility of each notification (Phase 1). As the participant browsed the page, each notification appeared in a timed sequence in order from least interruptive (i.e. Bar notification) to most interruptive (i.e. Dialog Box). Participants next completed a survey about whether they noticed each of the notifications when triggered.

Participants revisited each of the notification designs in the same order as deployed (Phase 2) and were asked to click on a specific link that triggered each notification individually. After triggering each notification, participants were asked to complete a survey noting their opinion of different aspects of each of the notification designs (e.g. familiarity, language used), and verbally elaborate on their questionnaire responses. After viewing all three notifications and interacting with them individually, participants were asked about their overall perceptions of the notifications they saw.

In Phase 3, participants were interviewed about their current pointing information needs and preferences. We asked participants to consider different types pointing information (e.g. performance data, manual instruction to improve pointing) they would want to receive from an adaptive pointing system and how they would use this information in their daily tasks. While we provided participants with specific examples of pointing information to help guide the interview, we encouraged participants to also think broadly about the types of information they might like to receive.

In the latter part of the interview (Phase 4), we transitioned to participants expectations for pointing assistance activation. Participants used the prototype to provide participants with examples of three types of assistance activation modes (i.e., manual, automatic, and mixed-initiative [11, 33]). Finally, participants noted on a survey their initial attitudes toward each type of assistance activation and selected and explained their preferred assistance activation mode.

\subsubsection{Analysis Procedures}

We performed a thematic analysis of qualitative data: responses to interview questions and self-reported, open-ended questionnaire responses to look for recurring themes. From this analysis, themes emerged regarding participants' views of how they would like to interact with an adaptive pointing system. Closed-ended questionnaire responses were analyzed with descriptive statistics.

\section{Findings and Design Recommendations}

Data from the study provided several insights into users' expectations and preferences for adaptive pointing systems. Three main themes emerged from the qualitative data analysis regarding users' initial preferences and expectations of adaptive pointing solutions and the rationale behind them. Users expected that the system 1) recognize user goals and adapt accordingly, 2) provide them with transparency about its functionality, and 3) put them in control of the interaction. We discuss each theme below.

\subsection{Recognizing Individual Goals and Adapting to Each Specific User}

Most of our participants were confident that an adaptive system could provide accurate automatic assistance $(85 \%)$ and deliver them quickly (70\%). They believed that such a system could correctly help complete a pointing task and provide that help efficiently. However, participants had concerns that extended beyond precision and correctness to whether a system could be sensitive enough to recognize and adapt to their unique goals.

Participants were hesitant about trusting an adaptive system that could be receptive of their unique preferences, provide them with needed support, and at the same time avoid adding more unpredictability and inconsistency to their experience. They were concerned if the system could recognize their goals and make changes in a way they felt it should. For example, P5 questioned whether her goals and the system's goals would be the same: "Is it going to try to second guess me? It is hard to predict something when you are not sure what it is like. On the iPad, it changes words ... that frustrates me. What's to say it doesn't adjust the way I want?" P4 also mentioned a similar concern; he stated, "I would grow in confidence [with a system] if it could demonstrate that it could make the right changes. It would increase my confidence ... the software could help maintain confidence if it had a history of doing things right." Younger adult participants expressed this attitude as well. When asked about how she felt about automatic pointing assistance, YA3 stated, "I don't know ... Because I don't know how it will determine what size adjustment I want.... maybe it will learn that I prefer $90 \%$ over $80 \%$ after a matter of time." In each of these cases, participants discussed the need for the system to take into consideration how they would personally like the pointing task completed. Therefore, participants expected that the system would assist them, and consider their unique goals for how they would like the pointing task completed.

Similar concerns emerged when discussing assistance activation. The majority of participants $(78 \%)$ preferred a mixed-initiative approach where they were informed about assistance (such as a change in interface) before it was activated and had the chance to confirm the change before it took place. When discussing their preferences for mixed-initiative approaches, one concern that emerged repeatedly as reasoning for this preference was whether the system could be flexible enough to meet expectations of their preferred interactions. For example, when asked about her preference for mixed-initiative, OA5 stated, "You feel like you have a little more control, and there is less room for interpretation". In this case, OA5 was concerned about a system's ability to accurately interpret how she would like the pointing task completed. Therefore, while quantitative data suggests that most participants believed an adaptive pointing system could help them complete their pointing task accurately and in a timely manner, they were less convinced that the system could successfully interpret their expectations of how the task should be completed.

Design Recommendations: These results indicate that an adaptive pointing system should put mechanisms that in place to gain the trust of users over time by recognizing and supporting user preferences for how the pointing task is completed. Since users that experience variable pointing are undergoing change that they themselves sometimes have difficulty predicting, it is reasonable for them to question whether an automatic system can respond correctly to their changing abilities.

We believe that consistently accurate performance, accompanied by thoughtful integration of unique preferences in the system may help gain the trust of its users. Initially such a system may be conservative in the notifications and assistance it provides as it learns more about the user. As more data is collected, the system may present more automatic predications. Informing the user about what data that the system is using can help build trust that the system is adapting specifically to their unique performance 
and not another group of users. Doing so can further maintain users' perceptions that the system supports individual goals.

\subsection{Providing Transparency for Decision- Making}

Participant concerns about trust also extended to the amount of information an adaptive pointing interface provided related to its actions. They wanted to system to provide transparency and summarize the kinds of errors the system detected and in what contexts. Participants also preferred to know why a particular type of assistance was recommended.

None of the participants liked the abstract indicator notification design and everyone stated that it provided inadequate information. Instead, most participants preferred the Dialog Box $(63 \%)$ or Bar+ $(30 \%)$ notifications not because of their designs, but because they provided more information and obvious support. When asked about the abstract indicator design, OA1 stated: "Not sure what the red line [abstract indicator] means. ... always nice to have an [immediate] explanation why something appeared." On the other hand, when discussing the dialog box design, participant OA10, "I like the fact that it tells you something is wrong. It doesn't tell you what you did, it just comes on and lets you know it is something. I would prefer it tells me something about what's wrong." Most participants were in favor of a system that provided information that could help them understand why it acted in the way that it did. For example, as OA10 wanted to know more about what led the system to determine that "something is wrong." Participants felt that having additional information could not only help them understand a system's actions, but also grow confidence in those actions.

Participants also valued information that could help them make informed decisions. P1 was not interested in immediate pointing performance information but wanted information that could help him make informed decisions about whether to activate recommended assistance. Specifically, he wanted to know why assistance was recommended and who or what made the recommendation, and like OA1 felt the abstract indicator notification was too vague "[It] doesn't work for me. [I] want to know everything about it immediately. [I] don't want to have to digfor information ... It doesn't tell me who is doing this. [I want] more information about why I am listening to you [the notification]." P2 thought by providing more information the system could make her computing experiences less frustrating. When asked about receiving pointing information, she said, "I think it would be helpful. I think it would make it less frustrating because I would know it is a motor impairment and not the computer. If I know that's what the issue is, I can focus on that and how to improve it." Participants wanted help with a decision whether to accept the information and how to move forward.

Design Recommendations: Overall, our results suggest that participants wanted the system to provide information about its recommendations and preferred to have some transparency in the way the algorithm adapted to their performance. They felt that receiving no pointing information or limited abstract notifications that "something occurred" were less useful for understanding system functionality or for helping them to decide what assistance to deploy. Again, users with variable pointing may have difficultly predicting when and how changes in pointing performance will occur. Therefore, it is understandable that they may question how a system determines that assistance is needed and/or how the recommended assistance will help them. Considering and supporting these information needs in the interface design can build a user's understanding of their actions in relation to the system's recommendations. Providing this level of transparency may also empower users to make informed decisions regarding the amount and type of support they might want from and adaptive pointing interface.

\subsection{Putting Users in Control}

Due to concerns about automatic assistance, participants' preference for self-governed decision-making (i.e. users making the ultimate decision to activate assistance) support was consistent across all groups. All participants wanted to be involved in the process of determining if a pointing assistance was activated and ultimately deployed. Of the three types of activation assistance discussed, the majority of participants $(78 \%)$ preferred a system that would provide a mixed-initiative approach that allowed them to review and confirm suggested assistances. Generally, participants were concerned about balancing automatic assistance with their individual preferences for maintaining control of their actions. Additionally, they believed that while automated assistance can correct errors, it is not always something that they wanted to utilize. For example, one participant provided the example of "predictive text" when typing and mentioned that while it does correct mistakes it can be annoying when it makes corrections when they are not wanted.

Most of the participants in the study preferred receiving both notification and assistance instead of notification or assistance alone. Because of concerns about automatic assistance, preference for self-governed decision-making (i.e. users making the ultimate decision to activate assistance) support was consistent across all groups. All participants wanted to be involved in the process of determining if a pointing assistance was activated and ultimately deployed. Preferences for notification design were also influenced by participants desire to be involved in assistance activation. Another reason the abstract indicator was participants' least favorite design was due to the fact that assistance activation was not available. Both the dialog box and Bar+ designs were favored because they both provided users with the choice to activate assistance or dismiss it. Participants did not trust that the system would activate the assistance in the way they expected and therefore, part of their preference for mixed-initiative was due to the fact that they felt it provided more control over the interaction. In this way, participants wanted to be able to review and correct possible deviations from their expectations that were automatically made by the system.

When asked about his initial reactions to a system that could assist with pointing, P4 stated, "[The] system could help in the area because maybe something may not make sense, but I would prefer if it let me do it and hold my hand." YA8 preferred a mixedinitiative approach, "[I would] prefer something hybrid because I can't see how a computer could intuit my needs accurately. Even then, I would prefer small, incremental changes. If it were to be automatic, I would be ok with ONE, small automatic one, and past that the computer asks me about changes." OA4 provided similar preferences for mixed-initiative activation, stating "Maybe I might like what I already have and I wouldn't want to change it. So, it [the system] gives me a choice." Participants preferred a system that included them in the decision-making process by allowing them to govern whether the automated assistance was deployed, collected feedback on whether the deployment met their needs, and adjusted the assistance based on this feedback.

Design Recommendations: Our results suggest that an adaptive system designed to support users with pointing difficulties should allow them to review and confirm assistance deployment. For users with unpredictable and/or varied pointing, allowing them to 
review changes and govern decisions can make them feel that they will ultimately maintain control of their interactions with the system (opposed to the system making decisions on their behalf). It can also be useful for a system to allow users access to the data that the system has collected from them and allow them to correct mistakes or specify which parts of the data to include or exclude in informing system functionality. By doing so, an adaptive pointing system can further increase transparency and build user confidence by including them in the decision-making process.

\section{PINATA: Demonstrating Design Recommendations for an Adaptive User Interface for Web Navigation}

Our findings indicate that meeting users' expectations for personalized pointing assistance extends beyond the act of providing accurate assistance. While accuracy was important to our participants, much of our findings suggest that it is also important for a system to also be able to recognize and respond to individual goals, provide transparency in its actions, and provide the user with adequate control of their pointing actions. It is therefore important for designers to acknowledge users' expectations for involvement in completing pointing tasks and accommodate this need in their design(s).

In this section, we demonstrate how we used our findings to inform the design of PINATA, an adaptive user interface for web navigation. We provide a framework of design considerations that help designers provide transparency and control of system actions.

\subsection{A Design Framework for Adaptive Pointing}

In Figure 2, we provide a framework of design considerations that summarize our findings and describe how to design systems that can keep users informed (KUI), put the user in control (PUC), and help the user trust the system (HUTS). Our findings suggest that these three considerations are important to understanding user goals, providing transparency, and control of pointing tasks.

\begin{tabular}{|c|c|c|c|}
\hline \multicolumn{4}{|l|}{ HUTS: Help Users Trust the System } \\
\hline \multicolumn{4}{|l|}{ PUC: Put the User in Control } \\
\hline \multicolumn{2}{|l|}{ KUI: Keep Users Informed } & & \\
\hline $\begin{array}{l}\text { 1. Describe what occurred and provide rationale for } \\
\text { recommended assistance }\end{array}$ & $x$ & & $x$ \\
\hline $\begin{array}{l}\text { 2. Provide information to support informed decision- } \\
\text { making about activation }\end{array}$ & $x$ & $x$ & \\
\hline $\begin{array}{l}\text { 3. Provide a preview of how the automated task will be } \\
\text { completed. }\end{array}$ & & $x$ & \\
\hline $\begin{array}{l}\text { 4. Provide alternative activation options but also } \\
\text { provide a way for users to opt out of assistance }\end{array}$ & & $x$ & \\
\hline $\begin{array}{l}\text { 5. In addition to accuracy and performance, consider } \\
\text { users' individual preferences for task completion }\end{array}$ & & $x$ & $x$ \\
\hline 6. Understand and incorporate users' expectations & & $x$ & $x$ \\
\hline $\begin{array}{l}\text { 7. Consider users expected level of automation and the } \\
\text { desired level of involvement in activation }\end{array}$ & & & $x$ \\
\hline $\begin{array}{l}\text { 8. Understand and incorporate users' expectations for } \\
\text { autonomy in task completion }\end{array}$ & $x$ & $x$ & $x$ \\
\hline
\end{tabular}

Figure 2. Recommended design considerations for personalized pointing system design.
Our participants wanted the systems actions to be transparent to assist them with making decisions about whether or not they wanted to activate assistance. Keeping users informed of system actions can help them better trust the system as well as help them feel more in control of the pointing task. Participants wanted to be involved in the activation of assistance therefore, recognizing users' individual goals and adapting the system in a way that supports those goals can also help users trust the system. We believe that the more the users trust the system to integrate their personal goals into its actions and meet their personal expectations, the more likely the user will be to adopt the system.

The three categories we present are interrelated and therefore, several of these design considerations traverse categories. To help solidify our recommendations, in the next section, we demonstrate how we applied these recommendations in the design of a webbased adaptive interface to support pointing.

\subsection{Designing PINATA}

To demonstrate how our findings, inform adaptive user interface design, we applied the recommendations to design, Pointing Interaction Notifications and AdapTAtions (PINATA). This system is an interactive adaptive interface for web navigation for users who experience pointing difficulties. It is implemented as a Chrome extension that tracks user pointing performance over time and in accordance with user-specified parameters adjusts system functionality to match a user's changing performance over time. It was built as an extended implementation of the prototype used in the user study.

PINATA has four components that work closely together to provide a user with notifications and assistance based on their pointing performance. The User Preferences Manager (1) provides users with an overview of the system and allows them to configure the type of notification and assistance they would like to receive. The Pointing History Browser (2) allows users to view their pointing performance using a series of visualizations. During the interaction, the user can activate assistance in the form of a customized Adaptive Bubble Cursor (3) that dynamically adjusts to user pointing performance. Finally, the user can enable Interactive Notifications (4) that inform them about pointing errors and provides them with the option of activating assistance. In the following subsections, we describe these modules and how their design is informed by user preferences and expectations identified in the previous section.

\subsection{User Preferences Manager}

The user can customize and control the functionality of PINATA through the User Preferences Manager which provides a series of options on how to deliver notifications and assistances (Figure 3). The user can tweak the functionality of the assistance (described in Section 5.3), preview and specify the type of notifications deployed when errors are detected (section 5.4) and allow the user to browse their pointing performance history (described next). This feature was included based on the results suggesting that users preferred options that would allow them to customize the system to their unique preferences (as described in Section 4.2 and Section 4.3). By allowing for customization, we hope to improve users overall trust that the system will adapt in a way they expect and also, meet their unique goals and preferences. 


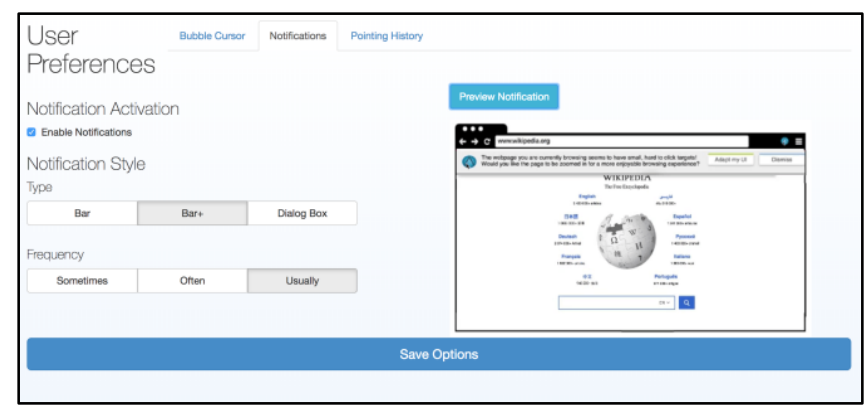

Figure 3. User Preferences Manager: This panel provides access to several pages (one example shown above) that let users preview and select various options with respect to PINATA's functionality. It allows users to specify the style and frequency of notifications and assistances and review their pointing history.

\subsection{Interactive Notifications}

PINATA provides users with the option to receive interactive notifications when pointing errors are detected. The user can specify notifications to appear as Bart or Dialog Box implementations that display information about the number and type of errors detected. All notifications present the user with a choice to deploy assistance or ignore them (in accord to findings described in Section 4.1). The user can choose from three levels of error frequencies that would trigger notifications (i.e., "sometimes", "often", "usually") in the User Preference Manager. For example, if a user selects to be notified if errors are "sometimes" detected, the system would notify them if on average one error is detected every five minutes during interaction. For the other conditions, more frequent errors will activate a notification. These values can be customized for each user. Currently, these notifications are consistent across all error types; in the future, we will provide the user with more notification formatting and deployment frequency options, including the choice to assign different notifications to different types of detected errors.

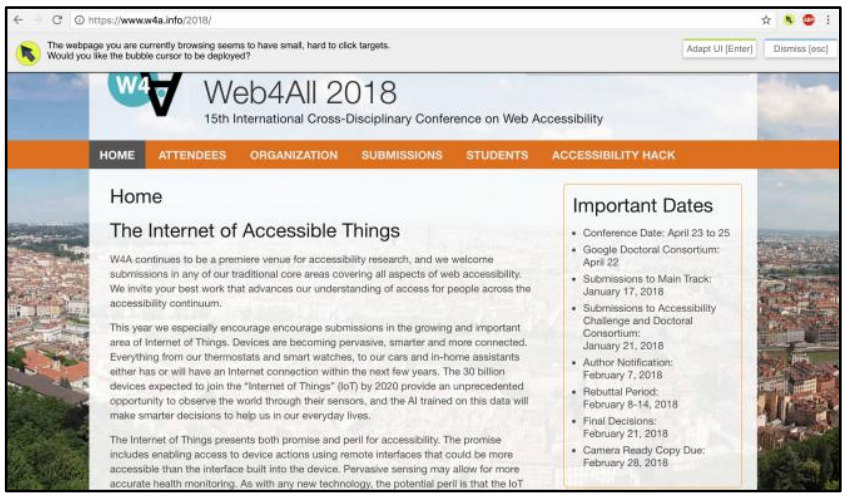

Figure 4. Interactive Notifications: Based on their preference, users are presented with prompts informing them when pointing errors happen. When a notification is deployed, users can ignore it, dismiss it, or activate assistance.

\subsection{Pointing History Browser}

The Pointing History Browser consists of two dynamic graphs that visualize the pointing performance of a user over time and based in the context they occur. Figure 5 shows the two graphs: The Error Type Graph shows the frequency and type of pointing errors that occurred over time. Currently, we are showing information about four types of common errors on this graph: slipping off target, missing target, unintentional selection. The
Website Graph shows the top 4 websites that pointing errors occurred on.
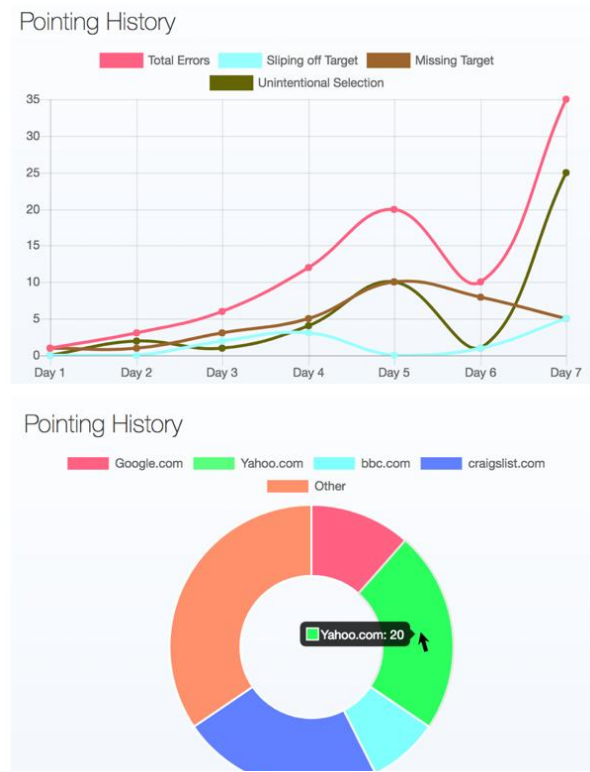

Figure 5. Pointing History Visualizations: Two types of visualizations are provided to the user. The Error Type Graph (top) shows the frequency of different kinds of error types over time. The Website Graph (bottom) shows the most common websites where pointing errors have occurred. When users hover over data points, additional information is shown.

This module serves two purposes: 1) it provides longitudinal feedback to the user about the type of errors that happened and 2) it allows them to view the underlying data that the system is using and correct and edit them if desired. Thus, this module addresses the preferences identified in the user study by supporting system transparency and allowing users to be in control.

Users can view the data in the graphs and delete their pointing performance history. However, users might also be interested in viewing and editing the underlying data that is collected to further increase user control and system transparency.

\subsection{Adaptive Bubble Cursor}

PINATA provides the user with dynamic assistance using an Adaptive Bubble Cursor. A bubble cursor dynamically resizes its selection area to assist target selection [8]. By increasing its size, it effectively decreases the precision needed to select targets. The increased selection area can either be visible or invisible a setting in the User Preferences Manager. Figure 6 shows a bubble cursor in action. We chose the bubble cursor as the assistance to provide because 1) previous research has shown that it can improve target selection for older adults [6, 27], and 2) it does not impact the layout of the underlying webpage while providing assistance. These features address our findings (described in Section 4.1 and Section 4.3) that showed a negative attitude in users towards unpredictable and major changes to the layout during interaction.

PINATA allows users to activate the assistance in two modes: manual or adaptive. In the manual mode, the user activates and deactivates bubble cursor whenever they need it. This is in effect the same as the original implementation of bubble cursor [8]. In the adaptive mode, user pointing performance is monitored and the selection size of bubble cursor is increased dynamically in response to an increase in the frequency of errors: the more errors, the larger the selection area of the cursor. In both modes, the user 
can specify the color and style of the cursor and whether its selection area is shown or is invisible.

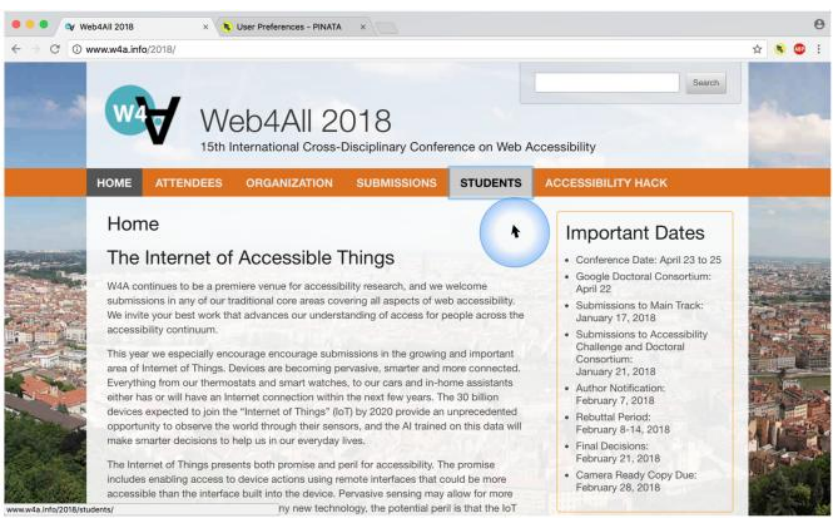

Figure 6. The Adaptive Bubble Cursor in action. The cursor's selection area grows in response to an increase in detected pointing errors. The larger size makes it easier to select objects close to the cursor. In the adaptive mode, the size and selection area of bubble cursor change in response to pointing error detection. The user can specify whether the selection area of the bubble cursor should be visible or hidden.

\section{DISCUSSION AND CONCLUSIONS}

Adaptive or personalized systems that monitor user activity and change their functionality accordingly are prevalent, especially on the Web. We conducted a user study to understand how to design an adaptive system that supports users who experience pointing difficulty due to intermittent or gradual decline in hand mobility. We found that while participants believed that such systems could help them, they were all hesitant about whether an adaptive pointing system would function according to their expectations and preferences. Participants wanted a system that would 1) introduce assistance slowly and adapt to their behavior over time while taking into account their unique preferences, 2) keep them informed about system functionality and actions, and 3) put them in control of the interaction. Using our findings, we present a framework of design considerations for personalized pointing systems. We demonstrate how our findings can be applied to adaptive interface design by discussing the design of PINATA, an adaptive interface that provides notifications and assistance to users when they experience pointing difficulties while browsing the Web. We described how PINATA allows users to view and control their pointing performance history, automatically or manually activate a bubble cursor to assist with pointing tasks and specify the type and frequency of notifications they would like to receive.

Our research emphasizes factors beyond performance and accuracy that can impact user perception and acceptance of personalized systems and complements other research on personalized systems that aim to support users who are undergoing changes in their abilities. Much of our findings indicate that understanding and integrating support for individual user goals as well as understanding and supporting individual user preferences for involvement in the pointing task is important for encouraging adoption. Adaptive and automated system research highlights the need to understand user preferences and expectations throughout the design process in order to design systems that are usable and encourage sustained use [16, 19]. Our research therefore contributes to a better understanding of the preferences and expectations of specific group of users that may benefit from an adaptive pointing interface. We also extend prior work that focuses on adaptive pointing system design by highlighting factors of the design that may encourage adaption.

\section{LIMITATIONS AND FUTURE WORK}

Our study, contributes a better understanding of users' expectations and preferences of adaptive pointing solutions. One limitation of our study is that our findings are based on a user's one-time interaction with a prototype interface. Although our study provides insights on initial expectations that may impact initial adoption of the solution, additional longitudinal studies with a fully implemented system will be needed to determine whether these preferences remain or change over time.

We focused on the process of designing PINATA and describing how its functionality is informed by user input. In a future study, we will evaluate the system in a longitudinal study where we examine users' perceptions and attitudes towards it and its impact on their Web navigation experience. We also expect to continue to refine the framework of design considerations based on these sets of studies.

This study focused on individuals who experience intermittent or gradual changes in hand mobility; other groups with severe or permanent motor impairments such as Cerebral Palsy, advanced Parkinson's, or Muscular Dystrophy, may have different preferences and expectations. Since we focused on notifications and assistance activation, we recommend future work explore user expectations about the deactivation of assistance or how their perceptions change over time.

While this study focused on pointing with a mouse, we anticipate these findings to be useful for other input devices. Future work is needed to understand how these results apply to touch and gesture, and interactions beyond the desktop including situational impairments with mobile device use.

\section{ACKNOWLEDGMENTS}

We would like to thank our participants for their input. This project was funded by a NIDILRR grant \#-90DP0061-01-00.

\section{REFERENCES}

1. Ahlström, D., Hitz, M., and Leitner, G. 2006. An evaluation of sticky and force enhanced targets in multi target situations. In Procs. of NordiCHI'06. ACM Press, 58-67.

2. Bunt, A., Mcgrenere, J., \& Conati, C. (2007). User Modeling 2007. (C. Conati, K. McCoy, \& G. Paliouras, Eds.) (Vol 4511). Berlin, Heidelberg: Springer Berlin Heidelberg. doi:10.1007/978-3-540-73078-1

3. Chaparro, A., Bohan, M., Fernandez, J., Choi, S. D., and Kattel, B. 1999. The impact of age on computer input device use: Intl. Journal of Industrial Ergonomics, 24(5), 503-513.

4. Chellappa, R. K., \& Sin, R. G. 2005. Personalization versus Privacy: An Empirical Examination of the Online Consumer's Dilemma. Information Technology and Management, 6(2-3), 181-202.

5. Diehl, M., Hooker, K., and Sliwinski, M. J. 2014. Handbook of intraindividual variability across the life span. London, UK: Routledge.

6. Findlater, L., Jansen, A., Shinohara, K. Dixon, M., Kamb, P., Rakita, J., and Wobbrock, J. O. 2010. Enhanced area cursors: reducing fine pointing demands for people with motor impairments. In Proc. of UIST '10. ACM Press, 153-162.

7. Gajos, K. Z., Weld, D. S., and Wobbrock, J. O. 2010. Automatically generating personalized user interfaces with Supple. Artificial Intelligence, 174:12-13, 910-950.

8. Grossman, T., Balakrishnan, R. (2005). The bubble cursor: enhancing target acquisition by dynamic resizing of the 
cursor's activation area. In Proc. CHI'05, ACM Press, 281 290.

9. Gregor, P., Newell, A. and Zajicek, M. 2002. Designing for dynamic diversity: interfaces for older people. In Proc. ASSETS'02. ACM, New York, NY, USA, 151-156.

10. Heron, M., Hanson, V., and Ricketts, I. 2013. Accessibility Support for Older Adults with the ACCESS Framework. International Journal of Human-Computer Interaction, 29(11), 702-716.

11. Horvitz. E. 1999. Principles of mixed-initiative user interfaces. In Proc. of CHI '99. ACM Press, 159-166.

12. Hourcade, J. P., and Berkel, T. R. 2008. Simple pen interaction performance of young and older adults using handheld computers. Interacting with Computers, 20(1), 166-183.

13. Hutchinson, H., Mackay, W., Westerlund, B., Bederson, B, Druin, A., Plaisant, C, Beaudouin-Lafon, M., Conversy, S., Evans, H., Hansen, H., Roussel, N., and Eiderbäck, B. 2003. Technology Probes: Inspiring Design for and with Families. In Proc. of CHI03, ACM Press, 17-24.

14. Hurst, A., Hudson, S. E., Mankoff, J., and Trewin, S. 2013. Distinguishing users by pointing performance in laboratory and real-world tasks. ACM Transactions on Accessible Computing, 5(2), 5 .

15. Hurst, A., Hudson, S. E., Mankoff, J., and Trewin, S. 2008. Automatically detecting pointing performance. In Proc. of IUI '08. ACM, 11-19.

16. Jamerson, A. 2008. Adaptive Interfaces and Agents. In A. Sears \& J. A. Jacko (Eds.), The human-computer interaction handbook: Fundamentals, evolving technologies and emerging applications (2nd ed.), 433-458. Boca Raton, FL: CRC Press.

17. Keates, S. and Trewin, S. 2005. Effect of age and Parkinson's disease on cursor positioning using a mouse. In Proc. of ASSETS'05. ACM Press, 68-75.

18. Ketcham, C. and Stelmach, G., 2004. Movement Control in the Older Adult. In RW Pew \& SB Van Hemel (Eds.), National Research Council (US) Steering Committee for the Workshop for Technology for Adaptive Aging, National Academies Press, 64-92.

19. Lavie. T. and Meyer. J. 2010. Benefits and costs of adaptive user interfaces. Int. J. Hum.-Comput. Stud., 68 (8), 508-524.

20. Li, L., Gajos, K. Z. 2014. Adaptive click-and-cross: adapting to both abilities and task improves performance of users with impaired dexterity. In Proc. of IUI '14. ACM Press, 299-304.

21. Majmudar, M. D., Colucci, L. A., and Landman, A. B. 2015. The quantified patient of the future: Opportunities and challenges. Healthcare (Vol. 3).

22. Martin-Hammond, A., Ali, A., Means, C., Hornback, C., and Hurst, A. 2016. Supporting Awareness of Pointing Behavior among Diverse Groups. In Proc. of PervasiveHealth. ACM Press, 231-234.

23. Martin-Hammond, A., Ali, A., Hornback, C., and Hurst, A. 2015. Understanding design considerations for adaptive user interfaces for accessible pointing with older and younger adults. In Proc. of W4A '15. ACM press, Article 19, 10 pages.

24. McCrickard, S. D. and Chewar, C. M. 2003. Attuning notification design to user goals and attention costs. Commun. ACM, 46(3), 67-72.
25. McCrickard, S.D., Chewar, C. M., Somervell, J.P and Ndiwalana, A. 2003. A model for notification systems evaluation-assessing user goals for multitasking activity. ACM Trans. Comput.-Hum. Interact., 10 (4), 312338.

26. Moffatt, K. and McGrenere, J. 2009. Exploring Methods to Improve Pen-Based Menu Selection for Younger and Older Adults. ACM Trans. Access. Comput. 2, 1, Article 3, 34 pages.

27. Moffatt, K. and McGrenere, J. 2010. Steadied-bubbles: combining techniques to address pen-based pointing errors for younger and older adults. In Proc. of CHI'10. ACM Press, $1125-1134$.

28. Moffatt, K., Yuen, S. and McGrenere, J. 2008. Hover or tap?: supporting pen-based menu navigation for older adults. In Proc. of ASSETS'08, ACM Press, 51-58.

29. Nielsen, J. 1993. Usability engineering. Cambridge, MA: Academic Press.

30. Pak, R., and McLaughlin, A. C. 2010. Designing displays for older adults. Boca Raton, FL: CRC Press.

31. Rothrock, L., Koubek, R., Fuchs, F., Haas, M., and Salvendy, G. 2010. Review and reappraisal of adaptive interfaces: Toward biologically inspired paradigms. Theoretical Issues in Ergonomics Science, 3(1), 47-84.

32. Sears, A., Lin, M., Jacko, J. and Xiao, Y. 2003. When Computers Fade: Pervasive Computing and SituationallyInduced Impairments and Disabilities. In Proc. of HCII'03, 1298-1302.

33. Shneiderman, B. and Maes, P. 1997. Direct manipulation vs. interface agents. interactions 4, 6 .

34. Sloan, D., Atkinson, M., Machin, C., and Li, Y. 2010. The potential of adaptive interfaces as an accessibility aid for older web users. In Proc. of W4A'10. ACM Press, Article 35, 10 pages.

35. Taveira, A. D., and Choi, S. D. 2009. Review Study of Computer Input Devices and Older Users. Intl. Journal of Human-Computer Interaction, 25(5), 455-474.

36. Trewin, S. 2000. Configuration agents, control and privacy. In Proc. of CUU '00, ACM Press, 9-16.

37. Trewin, S., Keates, S., and Moffatt, K. 2006. Developing steady clicks: a method of cursor assistance for people with motor impairments. In Proc. of ASSET '06. ACM Press, 2633.

38. Wickremaratchi, M. M., \& Llewelyn, J. G. 2006. Effects of ageing on touch. Postgraduate Medical Journal, 82(967), 301-304.

39. Wobbrock, J., Kane, S., Gajos, K., Harada, S., and Froehlich, J. 2011. Ability-Based Design: Concept, Principles and Examples. ACM Trans. Access. Comput. 3, 3, Article 9, 27 pages.

40. Wobbrock, J., Fogarty, F., Liu, S., Kimuro, S., and Harada, S. 2009. The angle mouse: target-agnostic dynamic gain adjustment based on angular deviation. In Proc. of CHI'09. ACM Press, 1401-1410.

41. Wood, E., Willoughby, T., Rushing, A., Bechtel, L., and Gilbert, J. 2005. Use of Computer Input Devices by Older Adults. Journal of Applied Gerontology, 24(5), 419-438.

42. Worden, A., Walker, N., Bharat, K., Hudson, S. 1997. Making computers easier for older adults to use: area cursors and sticky icons. In Proc. of CHI'97. ACM Press, 266-271. 